\title{
Cultivation of arterial stiffness fields in the land of the rising sun
}

\author{
Hirofumi Tanaka' ${ }^{1}$ \\ Received: 18 July 2018 / Accepted: 13 August 2018 / Published online: 28 August 2018 \\ (c) The Physiological Society of Japan and Springer Japan KK, part of Springer Nature 2018
}

\begin{abstract}
Arterial stiffness increases with advancing age and is an important risk factor for cardiovascular disease. As the number of research studies investigating arterial stiffness has increased dramatically over the recent years, their scope has broadened from physiological investigations to clinical domains focused on risk predictions. A number of key research studies were conducted in Japan in the formative years; however, some of these studies are unknown to most and largely forgotten. In this mini-review, some of these key formative research studies conducted in Japan by Japanese investigators are re-introduced and highlighted to provide appreciation for their pioneering work conducted in the land of the rising sun.
\end{abstract}

Keywords Japan $\cdot$ Japanese $\cdot$ Arterial elasticity $\cdot$ Vascular function $\cdot$ Pulse wave velocity

\section{Introduction}

Large elastic arteries undergo progressive stiffening with advancing age and with the presence of various risk factors and disease states [1]. Owing to the accumulating clinical evidence surrounding arterial stiffness and its clinical outcomes, arterial stiffness has become an independent predictor of cardiovascular morbidity and mortality and an important target for prevention and treatment of subclinical cardiovascular diseases [14]. In addition to being an independent risk factor, arterial stiffening is accompanied by a number of hemodynamic and neural sequelae. For instance, stiffening of large elastic arteries, in which the arterial baroreceptors are located, reduces the ability of those reflexogenic areas to transduce signals and impair baroreflex sensitivity [12]. Additionally, arterial stiffening could act to elevate aortic impedance, decrease stroke volume, and contribute to reduced functional capacity often seen in older adults [3, 20]. More recently, arterial stiffness has been implicated in the pathogenesis of cognitive dysfunction and dementia [4].

Hirofumi Tanaka

hatanaka@austin.utexas.edu

1 Cardiovascular Aging Research Laboratory, Department of Kinesiology and Health Education, The University of Texas at Austin, 2109 San Jacinto Blvd, D3700, Austin, TX 78712, USA
Given the multiplicity of its clinically important associations with a variety of clinical conditions, it is not surprising that the number of research studies dealing with arterial stiffness increased markedly in recent years, particularly since 2000 (Fig. 1). Japan is one of the leading countries that have constantly contributed a large number of research studies to the field of arterial stiffness. Even prior to the surge in research activities in 2000, a number of ground breaking and pioneering research studies were conducted in Japan. However, many of these studies have not seen the light or remain relatively unknown for a variety of reasons (e.g., articles were written only in Japanese). This is unfortunate since the research studies that we conduct currently and in the future are built on the foundations that past research has constructed.

Accordingly, the primary aim of the present brief review is to introduce some of the key research studies in arterial stiffness conducted in Japan during the early formative years. In order to highlight and focus on key research studies, the inclusion of the research studies is selective in nature rather than being comprehensive.

\section{Methodological studies}

A number of different techniques and methodologies have been used to assess the elastic properties of arteries in humans. Among them, pulse wave velocity has been most frequently used as a measure of arterial stiffness, and has 


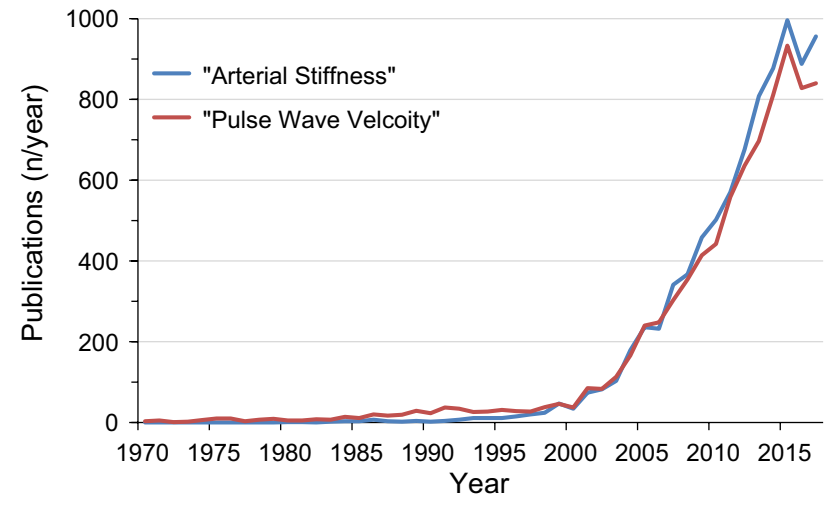

Fig. 1 The yearly number of research papers listed in PubMed using the key words of either "arterial stiffness" or "pulse wave velocity"

emerged as the reference standard in the field due to the accumulating clinical evidence [22]. Pulse wave velocity is calculated by the measurement of the pulse wave transit time and the distance between the two recording sites. A faster pulse wave velocity reflects stiffer arteries. Because the measurement of pulse wave velocity is simple, noninvasive, and reproducible, there are currently many commercial devices that are capable of measuring pulse wave velocity $[2,6]$.

It is not widely known that the world's first automated commercial device for measuring pulse wave velocity (and arterial stiffness for that matter) was manufactured and sold in Japan. In 1968, Fukuda Electro (the predecessor of the Fukuda Denshi) produced the Arterial Distensibility Counter KM-1 (Fig. 2A). This device utilized an ECG signal and peripheral arterial waveforms to measure the time delay between the heart and the peripheral artery measurement site. This original device was followed by the Mechano Cardiograph MCG-400 that was manufactured and sold in 1983 (Fig. 2B). This newer device incorporated the measurements of pulse waves at two different recording sites to assess the velocity of transmission of the pulse wave. Considering that the research studies in arterial stiffness did not take off till 2000 (Fig. 1), it is remarkable that a Japanese company had the foresight to capture and quantify arterial stiffness back in the 1960s.

In spite of accumulating evidence indicating the clinical importance of arterial stiffness, a measure of arterial stiffness has not been incorporated in routine clinical practice worldwide. However, there are a few countries in the world in which arterial stiffness is fully integrated into clinical medicine. One of the countries is Japan. This success has been mainly attributed to the development of brachialankle pulse wave velocity, which was originally developed by Colin Medical (the device is called Form in Japan or VP-1000 elsewhere) $[18,23]$. The most significant characteristic of this device is its ease of use, as the measurement
(A)

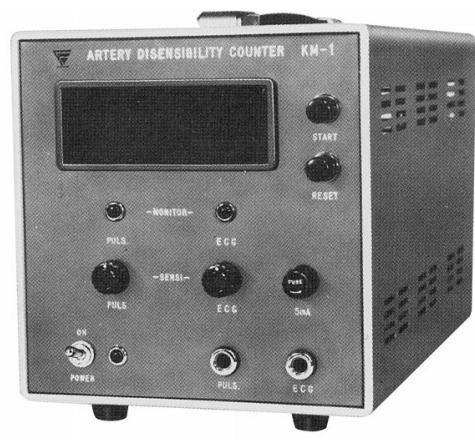

(B)

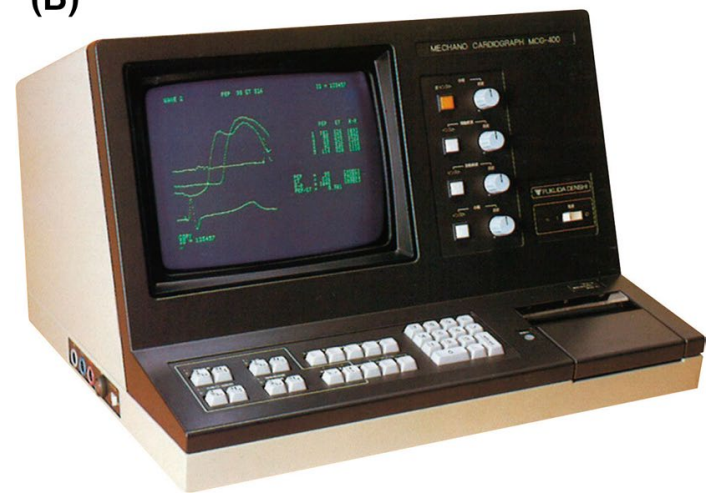

Fig. 2 Commercial devices manufactured by Fukuda Denshi to measure pulse wave velocity. A Fukuda Electro Arterial Distensibility Counter KM-1 that was manufactured and sold in 1968 and B Fukuda Denshi Mechano Cardiograph MCG-400 that was manufactured and sold in 1983. These pictures are shown here with the permission from Fukuda Denshi

can be done very quickly (in $5 \mathrm{~min}$ ) and, unlike other devices, does not require technical expertise. An impressive number of machines $(\sim 10,000)$ have been incorporated into various clinics and hospitals all over Japan. Substantial amounts of important clinical information have been generated by the network of Japanese investigators who utilized brachial-ankle pulse wave velocity [13].

Arterial stiffness or, more precisely, arterial compliance can also be derived from the simultaneous assessments of pulsatile changes in arterial diameter and pulse pressure using an imaging modality such as ultrasound. Because arterial stiffness is closely associated with arterial pressure and arterial pressure is an independent risk factor for cardiovascular disease, investigators have worked to create an index of arterial stiffness that is independent of arterial pressure. A pioneering index of arterial stiffness that incorporates a mathematical correction for blood pressure effects is the beta-stiffness index. This index has been used widely in the literature and has also served as a basis for newer indices of arterial stiffness, including Cardio-Ankle Vascular Index (CAVI) [16]. The idea of the beta-stiffness index was initially conceived by Hayashi et al., who studied static 
behavior of the arterial wall using intracranial and extracranial arteries obtained from autopsy samples [7]. Subsequently, a beta-stiffness index incorporating the logarithmic conversion of the ratio of systolic and diastolic blood pressure was established and applied to clinical samples by Hirai et al. [8]. Because this index characterizes the deformation behavior of the vascular wall independent of the intraluminal pressure within the physiological range, this index conceived by two different groups of Japanese investigators was very well received by fellow scientists in the field, and has contributed importantly to methodological refinements in the field [19].

\section{Physiological studies}

Arterial stiffness is primarily determined by the intrinsic elastic properties of the artery. The three primary elements of the arterial wall that determine its stiffness, particularly for large and moderate sized arteries are (1) the amount/ proportion of intimal-medial wall elastin and collagen (quantitative structural elements), (2) the cross-linking of collagen and advanced glycation end products (qualitative structural elements), and (3) the vasoconstrictor tone exerted by its smooth muscle cells (functional or vasoconstrictor elements). Among these, the role of endothelial function in modulating the vasoconstrictor tone and arterial stiffness has been intensively investigated [5, 17]. A common approach for determining endothelial function in humans is to assess vasodilatory responses to a pharmacological agonist for NO synthesis and release. Most often, acetylcholine has been used as an agonist.

It is virtually unknown to most that as early as 1940, Hirose investigated the effects of pharmacological infusion of acetylcholine on pulse wave velocity in ten human subjects [9]. He demonstrated that acetylcholine infusion reduced pulse wave velocity by increasing pulse transit time, and that the effects were independent of changes in heart rate [9]. Even though this study may be the first study to demonstrate the involvement of endothelial function on modulating arterial stiffness, the recognition and credit are currently lacking. This is probably due to the fact that the article was written in Japanese (and German) and that nitric oxide was not discovered till 40 years later.

\section{Prevention studies}

For most risk factors for cardiovascular disease, the firstline approach for prevention and treatment for the development of cardiovascular disease is lifestyle modifications [21]. Considering the important role that arterial stiffness plays as a precursor to cardiovascular disease, it is critical to determine the proper strategies to prevent and reverse arterial stiffening. One of the lifestyle modifications that has been widely investigated and found to be effective in vascular destiffening is regular aerobic (endurance) exercise [15]. Unknown to most, the first exercise intervention study for evaluating the effects of physical exercise intervention was conducted by Ikegami et al. back in 1983 [10]. This particular study was never published in English or any languages other than Japanese, but the details of the study should be introduced to the investigators in the field.

Ikegami et al. [10] studied a total of 80 apparently healthy young male military recruits and placed them in a physical training program consisted of calisthenics, jogging/running, swimming, soccer, and judo. These exercises were performed for an average of $2 \mathrm{~h}$ and $10 \mathrm{~min}(130 \mathrm{~min}) \mathrm{a}$ day, 6 days a week for 10 months (43 weeks). The efficacy of the exercise training program was demonstrated by significant improvements in a variety of physical fitness tests (e.g., $1500 \mathrm{~m}$ running, grip strength, trunk flexibility) implemented in the study. Aortic PWV decreased from $6.60 \pm 0.71$ to $6.27 \pm 0.61 \mathrm{~m} / \mathrm{s}$. Interestingly, greater reductions in aortic PWV were observed in those subjects with lower initial body fat levels (Fig. 3) and those who experienced larger changes in red blood cell mass [10]. Even though this study does not have a proper sedentary (non-exercising) control group to compare against, the exercise intervention was conducted very well with a long intervention duration (10 months) and a large number of subjects $(n=80)$. Ikegami et al. also conducted and published another exercise training study in 1983 that involved 70 middle-aged men in their 40s [11]. Exercise training was $90 \mathrm{~min}$ per session, 5 times a week for 4 months. Aortic pulse wave velocity decreased significantly in subjects as whole, and there were greater reductions in pulse wave velocity for those with higher baseline pulse wave velocity [11]. It is unfortunate that these excellent exercise interventional studies have not gathered any attention in the research field due to the language that these papers were written.

\section{Summary}

Tremendous progress has been made in the research field of arterial stiffness over the years. Papers published by Japanese investigators have made important contributions to the field by addressing many of the critical questions essential for our understanding of arterial stiffening. At the same time, there are a number of important research contributions that remain unknown to most. This is unfortunate because the progress of the field is built upon the foundation formed by the pioneers in the field. This review was written to highlight such efforts in order to encourage and facilitate future efforts by the next generation of Japanese investigators. 

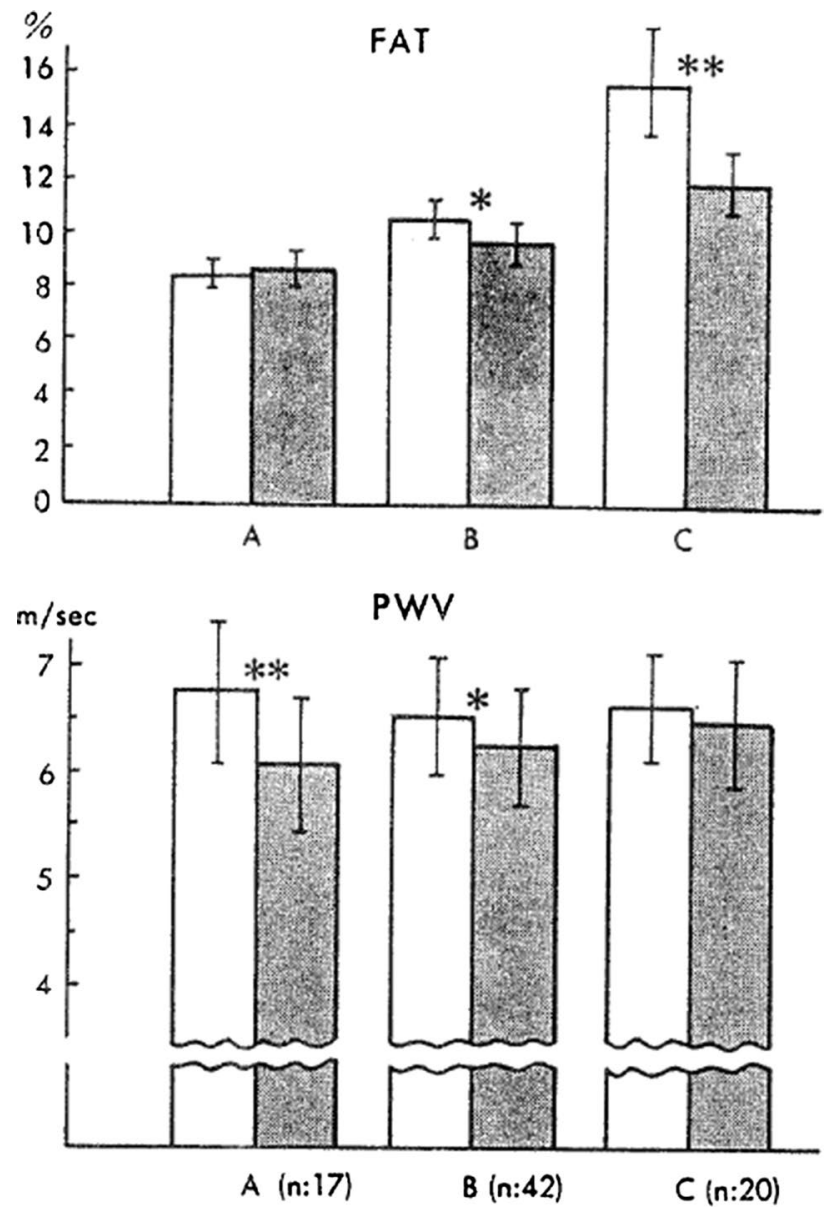

Fig. 3 Effects of the exercise intervention on arterial stiffness measured by aortic pulse wave velocity [10]. Open (white) and filled (black) bars are pre- and post-exercise intervention values. The subjects were divided into 3 different groups $(\mathbf{A}-\mathbf{C})$ based on the pretraining body fat levels. Effects of the exercise training intervention manifested in 2 lower body fat groups $(\mathbf{A}, \mathbf{B})$, whereas no significant effect was observed in the higher body fat group $(\mathbf{C})$. Reproduced with the permission from the publisher

\section{Compliance with ethical standards}

Conflict of interest The author declares that there is no competing interest.

\section{References}

1. AlGhatrif M, Lakatta EG (2015) The conundrum of arterial stiffness, elevated blood pressure, and aging. Curr Hypertens Rep 17:12

2. Asmar R, Topouchian J, Pannier B, Benetos A, Safar M (2001) Pulse wave velocity as endpoint in large-scale intervention trial. The Complior study. Scientific, quality control, coordination and investigation committees of the Complior study. J Hypertens 19:813-818

3. Chen CH, Nakayama M, Talbot M, Nevo E, Fetics B, Gerstenblith G, Becker LC, Kass DA (1999) Verapamil acutely reduces ventricular-vascular stiffening and improves aerobic exercise performance in elderly individuals. J Am Coll Cardiol 33:1602-1609

4. Cooper LL, Mitchell GF (2016) Aortic stiffness, cerebrovascular dysfunction, and memory. Pulse (Basel) 4:69-77

5. Correia ML, Haynes WG (2007) Arterial compliance and endothelial function. Curr Diab Rep 7:269-275

6. Cortez-Cooper MY, Supak JA, Tanaka H (2003) A new device for automatic measurements of arterial stiffness and ankle-brachial index. Am J Cardiol 91:1519-1522

7. Hayashi K, Handa H, Nagasawa S, Okumura A, Moritake K (1980) Stiffness and elastic behavior of human intracranial and extracranial arteries. J Biomech 13:175-184

8. Hirai T, Sasayama S, Kawasaki T, Yagi S (1989) Stiffness of systemic arteries in patients with myocardial infarction: a noninvasive method to predict severity of coronary atherosclerosis. Circulation 80:78-86

9. Hirose T (1940) Influence of medications on pulse wave velocity: part 1 effects of acetylcholine. Jpn Circ J 5:36-38

10. Ikegami H, Satake M, Kurokawa T, Tan N, Sugiura T, Yamazaki Y (1983) Effects of physical training on body composition, respiro-circulatory functions, blood constituents, and physical abilities. Part 1: men aged 30 years. J Phys Fitness Jpn 32:302-309

11. Ikegami H, Satake M, Seya K (1983) Effects of exercise training on arterial stiffness assessed by pulse wave velocity. J Phys Fitness Jpn 32:212-214

12. Monahan KD, Dinenno FA, Seals DR, Clevenger CM, Desouza CA, Tanaka H (2001) Age-associated changes in cardiovagal baroreflex sensitivity are related to central arterial compliance. Am J Physiol Heart Circ Physiol 281:H284-H289

13. Ohkuma T, Ninomiya T, Tomiyama H, Kario K, Hoshide S, Kita Y, Inoguchi T, Maeda Y, Kohara K, Tabara Y, Nakamura M, Ohkubo T, Watada H, Munakata M, Ohishi M, Ito N, Nakamura M, Shoji T, Vlachopoulos C, Yamashina A, Collaborative Group for JB (2017) Brachial-ankle pulse wave velocity and the risk prediction of cardiovascular disease: an individual participant data meta-analysis. Hypertension 69:1045-1052

14. Safar ME, Henry O, Meaume S (2002) Aortic pulse wave velocity: an independent marker of cardiovascular risk. Am J Geriatr Cardiol 11:295-298

15. Seals DR, Desouza CA, Donato AJ, Tanaka H (2008) Habitual exercise and arterial aging. J Appl Physiol 105:1323-1332

16. Shirai K, Hiruta N, Song M, Kurosu T, Suzuki J, Tomaru T, Miyashita Y, Saiki A, Takahashi M, Suzuki K, Takata M (2011) Cardio-ankle vascular index (CAVI) as a novel indicator of arterial stiffness: theory, evidence and perspectives. J Atheroscler Thromb 18:924-938

17. Sugawara J, Komine H, Hayashi K, Yoshizawa M, Yokoi T, Otsuki T, Shimojo N, Miyauchi T, Maeda S, Tanaka H (2007) Effect of systemic nitric oxide synthase inhibition on arterial stiffness in humans. Hypertens Res 30:411-415

18. Sugawara J, Tanaka H (2015) Brachial-ankle pulse wave velocity: myths, misconceptions, and realities. Pulse (Basel) 3:106-113

19. Tanaka H (2018) Various indices of arterial stiffness: are they closely related or distinctly different? Pulse (Basel) 5:1-6

20. Tanaka H, Dinenno FA, Hunt BE, Jones PP, DeSouza CA, Seals DR (1998) Hemodynamic sequelae of age-related increases in arterial stiffness in healthy humans. Am J Cardiol 82:1152-1155

21. Tanaka H, Safar ME (2005) Influence of lifestyle modification on arterial stiffness and wave reflections. Am J Hypertens 18:137-144

22. Van Bortel LM, Laurent S, Boutouyrie P, Chowienczyk P, Cruickshank JK, De Backer T, Filipovsky J, Huybrechts S, Mattace-Raso FU, Protogerou AD, Schillaci G, Segers P, 
Vermeersch S, Weber T (2012) Expert consensus document on the measurement of aortic stiffness in daily practice using carotid-femoral pulse wave velocity. J Hypertens 30:445-448

23. Yamashina A, Tomiyama H, Takeda K, Tsuda H, Arai T, Hirose K, Arai T, Hirose K, Koji Y, Hori S, Yamamoto Y (2002)
Validity, reproducibility, and clinical significance of noninvasive brachial-ankle pulse wave velocity measurement. Hypertens Res 25:359-364 